\title{
Hybrid lipid oligonucleotide conjugates: synthesis, self-assemblies and biomedical applications
}

\author{
Amit Patwa, ${ }^{a, b}$ Arnaud Gissot, ${ }^{a, b}$ Isabelle Bestel, ${ }^{a, b}$ and Philippe Barthélémy ${ }^{* a, b}$
}

Hybrid lipid oligonucleotide conjugates are finding more and more biotechnological applications. This short critical review highlights their synthesis, supramolecular organization as well as their applications in the field of biotechnology.

\section{Introduction}

Bioconjugates combining lipids and oligonucleotides (LONs) are attracting currently a considerable attention owing to their unique physicochemical and biological properties. Interestingly, these amphiphiles, which feature molecular recognition capabilities, the ability to store and transfer encoded information, self assemble to give aggregates such as micelles, ${ }^{1}$ liposomes ${ }^{2}$ and nanoparticles. ${ }^{3}$ In parallel, LONs have been developped for cell biology, and medicine ${ }^{4}$ as for example in the design of artificial molecular devices ${ }^{5-9}$ and novel therapeutic strategies. ${ }^{10}$

In this contribution we highlight recent advances in the area of amphiphilic structures derived from LONs with an emphasis on molecular and supramolecular properties, and biotechnological applications. In the first section, we focus on the design and the synthesis of LONs. This part includes several examples of synthetic oligonucleotide based amphiphiles. In the next section, we present the use of LONs as supramolecular building blocks and their aggregation properties. Finally, in the last section of this short critical review, we describe recent biomedical applications involving LONs. Figure 1 shows the capabilities of forming supramolecular assemblies and biomedical applications of LONs.

\section{Design and synthesis of LONs}

Development of efficient and reproducible methods for convenient preparation of various types of LONs has become a subject of considerable importance. Scheme 1 describes the possible ways of hydrophobic conjugations to the different moieties of oligonucleotide (i. e. at sugar, phosphate backbone or base unit). Hydrophobic part can be lipid (LONs) or fluorinated alykyl chain $(\mathrm{FONs})^{11}$ or any other surfactant (DNAsurf.).

Organic chemistry and solid phase synthesis (SPS) are unavoidable expertises required for the synthesis of LONs. Two general strategies exist for the incorporation of simple hydrophobic modification(s) anywhere (Figure 2A) in the sequence of the oligonucleotides, called presynthetic and postsynthetic approach (Figure 2B and 2C).

Presynthetic approach denotes that the nucleotide monomers already carry the desired hydrophobic moiety before oligonucleotide synthesis, deprotection, and purification. That

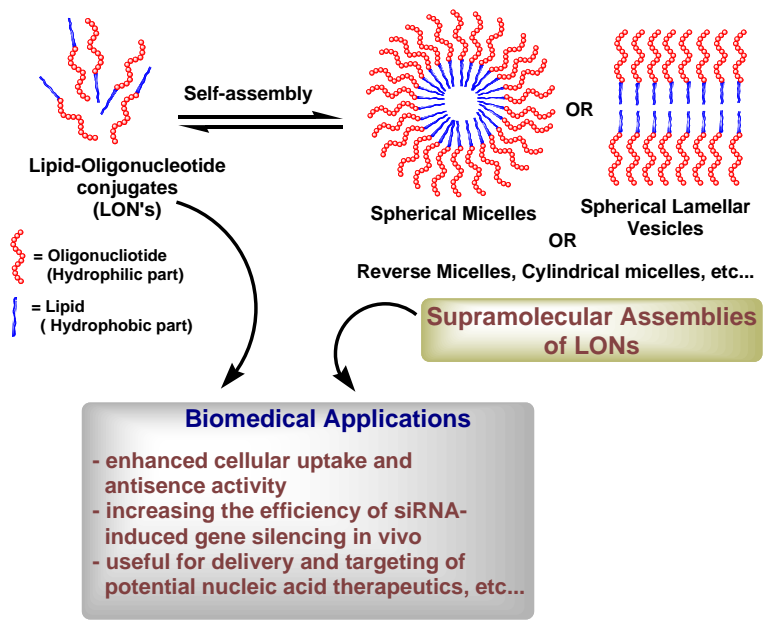

Figure 1. Lipid-oligonucleotide conjugates (LONs): building blocks for supramolecular applications and potential biomedical applications

is, these modified nucleotides are incorporated into the oligonucleotide sequence during the usual phosphoramidite process. Post synthetic labelling requires the introduction of a small reactive moiety into oligonucleotide, which can be coupled to the hydrophobic part after completion of oligonucleotide synthesis. The synthesis and properties of DNA block copolymers have been reviewed recently and the reader is referred to this article. ${ }^{12}$ Recent reviews highlight some examples of oligonucleotide based amphiphiles ${ }^{4}$ and the advances made in the solid support synthesis of oligonucleotide-lipid conjugates. ${ }^{13}$

\subsection{Presynthetic Approach: Hydrophobic modification during the oligonucleotide solid-phase synthesis}

In the presynthetic strategy a phosphoramidite bearing the desired hydrophobic moiety needs to be stable enough to survive the conditions employed during SPS (acidic and oxidative conditions) and deprotection (alkaline condition) of oligonucleotide chain. Thus, the wealth of chemical functions compatible with oligonucleotide SPS is restricted as this method excludes the use of hydrophobic moieties with reactive functional groups or inherent base- or acid-sensitivity. 


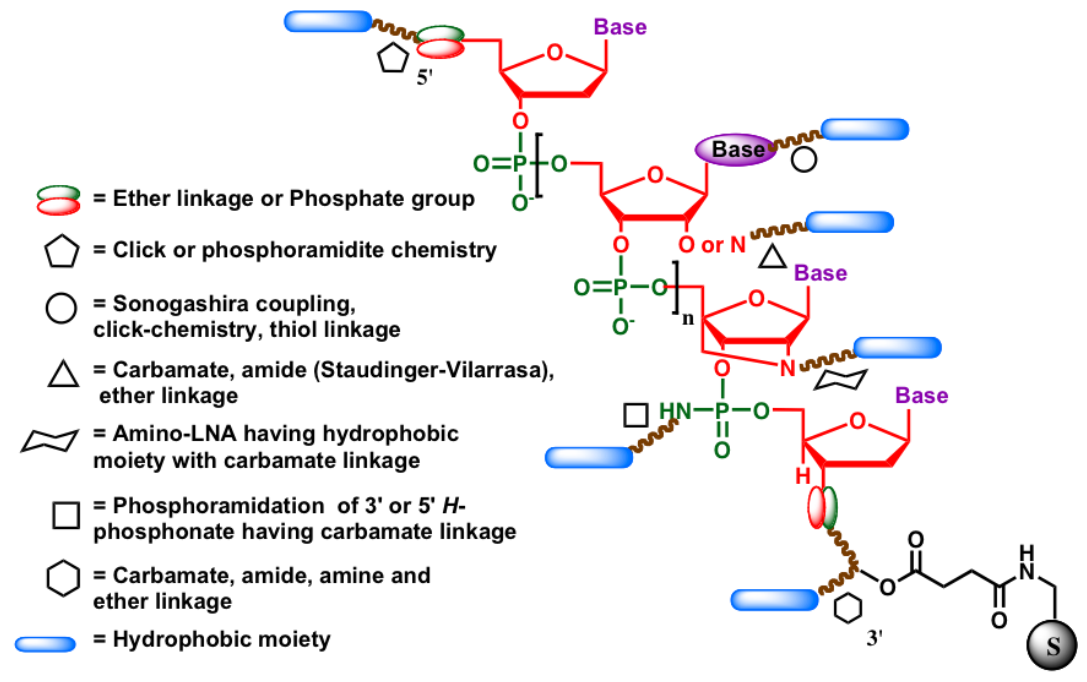

Scheme 1. Schematic representation for LONs showing different sites of hydrophobic conjugations.

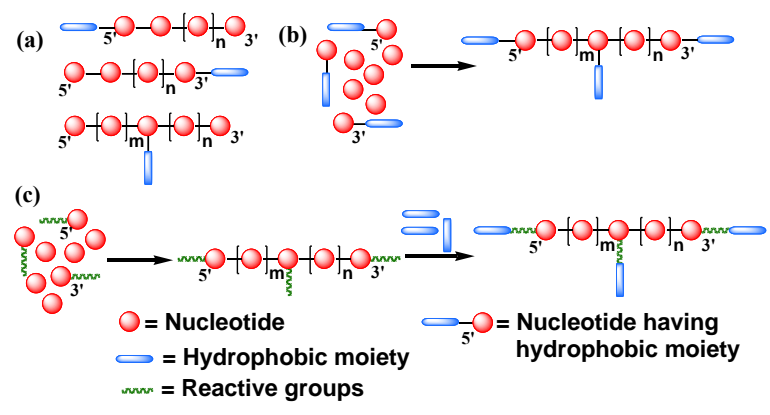

Figure 2. Schematic representation for (a) LON structures (Hydrophobic moieties can be incorporated either at the termini (3'and/or 5') or within the oligonucleotide sequence) (b) presynhetic approach (c) postsynthetic approach.

The hydrophobic conjugates can be incorporated usually at three different positions in oligonucleotide sequence: (i) $3^{\prime}$ modification by chain assembly on a solid support bearing the desired hydrophobic moiety, (ii) 5'-modification by introduction of the hydrophobic group as a phosphoramidite reagent, and (iii) intra-chain modification by making use of prefabricated nucleosidic building block or their congeners incorporating the conjugate group. It is more convenient to incorporate the hydrophobic modification at the 5 '-end compared to $3^{\prime}$-end as oligonucleotide chain extension proceeds in the $3^{\prime}-5^{\prime}$ direction. The modification at $3^{\prime}$-end requires substantial additional efforts in chemical synthesis. ${ }^{14,15}$ The first solid-phase synthesis of a cholesterol conjugate, developed by Letsinger is an elegant strategy to functionalize the 3 '-terminal, involving oxidative phosphoramidation of a support bound dinucleoside-3', 5 ' $-(\mathrm{H}-$ phosphonate). ${ }^{16}$ Interestingly, one of the recent approaches follows a similar principle. An aminoalkyl support, acylated with 3-chloro-4-hydrozyphenylacetic acid, was used instead of conventional succinyl linker. Later, $3^{\prime}$-terminal nucleoside is coupled to the hydroxyl function by the usual phosphoramidite chemistry followed by removal of the 2-cyanoethyl protection and coupling of the hydrophobic moiety to the phosphodiester linkage by the phosphotriester chemistry. The oligonucleotide sequence is then assembled by phosphoramidite chemistry. ${ }^{17}$ There are several other examples for the preparation of $3^{\prime}$ modified oligonucleotides. Here a branched alcohol having orthogonally protected amino and hydroxyl functionalities is coupled to the solid support via succinyl linker. The hydrophobic group having hydroxyl group is first coupled to the deprotected amino group as a chloroformate ester, and oligonucleotide chain is then assembled on the deprotected hydroxyl function. ${ }^{18,19}$ More often, related supports have been prepared by immobilization of prefabricated linkerhydrophobic conjugates. ${ }^{20-27} 3$ '-terminal modification can be achieved by another type of solid support linked to uridine either at $2^{\prime}-\mathrm{O}^{14}$ and $3^{\prime}-\mathrm{O}^{28}$ through succinyl linker and conjugated with hydrophobic moiety (via carbamate, amide or ether linkage) to the remaining secondary hydroxyl function. There are several phosphoramidite and $\mathrm{H}$-phosphonate reagents reported in the literature, both non-nucleosidic and nucleosidic ones, used to introduce hydrophobic moiety into the 5 '-terminus of oligonucleotide chains. ${ }^{15,25,26,28-32}$ Recently our group has utilized the Huisgen 1,3-dipolar cycloaddintion of alkynes and azides, ${ }^{33}$ a typical example of a "click" reaction, ${ }^{34}$ for modification of oligonucleotide conjugates at $5^{\prime}$-terminus. ${ }^{35}$ The use of ester linkage for conjugation of the hydrophobic moiety to the oligonucleotide is still restricted because of its cleavage during the final treatment with ammonia required for deprotection of the nucleobases and cleavage from the control pore glass (CPG) solid support. In fact, the majority of hydrophobic conjugates of oligonucleotides reported with ester linkages involves methods performing the conjugation step postsynthetically in solution. ${ }^{36-38}$ As a result, only polydT ester conjugates, where the base residue does not require protection, was fully synthesized on CPG. ${ }^{39,40}$ An alternative strategy for the deprotection and cleavage from the solid support has been devised that maintains the integrity of the ester bond. Here, 5'- 
modified oligonucleotide have been obtained by using baselabile $t$-butylphenoxyacetyl protecting groups for base moiety amino functions and oxalyl linker. These modifications to the standard phosphoramidite approach allow mild deprotection with ethanolamine, and hence, the 5'-ester linkage remains intact. ${ }^{40}$ 5'-Modification of CPG-bound oligonucleotides has also been carried out by elongation of the chain with 6chloroacetamidohexanol phosphoramidite and displacing the chlorosubstituent with thiocholesterol. ${ }^{41}$ Several lipophilic alcohols have been introduced at the 5'-terminus by first converting it into 2-alkoxy-2-thiono-1,3,2-oxathiaphospholane and then reacted with the 5'-hydroxyl group of the supportbound oligonucleotide followed by normal ammonolysis. ${ }^{42}$

Use of prefabricated nucleosidic or non-nucleosidic building blocks allows to introduce the hydrophobic moieties into interchain position of the oligonucleotide chain. Different approaches have been investigated for preparing hydrophobic phosphoramidite building block including 2'-derivatized uridine, ${ }^{4,28}$ derivatized heterocyclic bases, ${ }^{43-45}$ and a thymine amino-LNA monomer. ${ }^{46}$ Recently our group has reported an efficient and versatile access to a series of antagomir analogues. It described the synthesis of 2'-lipid-amido uridine phosphoramidite building block via Staudinger-Vilarrasa reaction. To the best of our knowledge, it is the first report of modified antagomirs featuring lipid moieties inserted withing the sequences ${ }^{47}$. Guzaev developed the phosphoramidite building block, derived from dioctyl 2,2bis(hydroxymethyl)malonate, whose aliphatic ester moieties are only slowly cleaved off by ammonia under the conditions routinely used for the deprotection of oligonucleotides thus allowing efficient multiple modification of oligonucleotides with hydrophobic moieties. ${ }^{48} \mathrm{Kim}$ et al. reported a novel phosphoramidite derived from lithocholic acid. With help of this phosphoramidite they were able to introduce hydrophobic segments in the middle of the oligonucleotide chain so that they would be mimics of hairpin structures. ${ }^{49}$

\subsection{Post-synthetic hydrophobic modification of oligonucleotides}

In a postsynthetic modification approach, a small reactive group is introduced into oligonucleotide chain, which can be then conjugated with the desired hydrophobic moiety in a selective manner after oligonucleotide synthesis and deprotection. Postsynthetic approach fall in two major categories: solution phase and solid phase hydrophobic modificaton. In solution phase approach, the oligonucleotide is synthesized in usual manner by SPS carrying a reactive functionality. The hydrophobic group is then coupled in solution after cleavage and purification of the oligonucleotide. While in solid-phase approach, the hydrophobic conjugate is assembled on a single support after the oligonucleotide synthesis. Kabanov et al. described an interesting protocol for the hydrophobization of 5'-phosphate oligonucleotides with lipophilic alcohol in surfactant reversed micelles in organic solvents. ${ }^{50}$ This method seems to be very promising in drug design, since it can be applied to any natural oligonucleotides having 5'-phosphate group and produced lipid modified oligonucleotides in high yields. Abell et al. developed a more classical approach for the derivatization of oligonucleotides with activated acid or aliphatic thiols in an aqueous buffered medium. ${ }^{51,52}$

\section{LON auto-organization and incorporation into membranes.}

As seen in the previous section, numerous strategies have been developed for the introduction of one or several hydrophobic motifs to oligonucleotides. Obviously, the lipophilic segments confer amphiphilic as well as self-aggregation properties to the resulting LONs. Yet, the self-assembly capabilities of LONs has often been overlooked in the past, especially in the case of antisense LONs. Moreover, it must be emphasized that investigation of the self-aggregation of LONs is still hampered by the difficulty in producing large quantities of these materials $(0.2$ to one micromole are synthesized in most reports).

Basically, LONs can be regarded as disymmetric, double-sided scotch tapes with 1) a poorly specific lipidic glue side that allows for the self-association and/or insertion of LON into membranes associated with 2) an oligonucleotidic smart side responsible for the specific recognition of defined targets (oligonucleotides or others). The synergy between the mode of recognition of the lipid and the oligonucleotide is central in the many recent applications of LONs published lately that will be emphasized in the last part of this section.

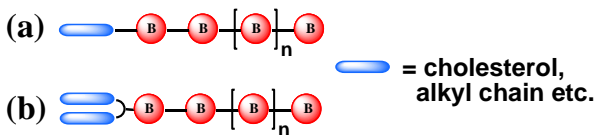

Figure 3. Schematic drawing of (a) a simple-chain and (b) a double-chain LON.

\subsection{Self-assemblies of LON}

LONs being mainly utilized in biotechnological contexts, their aggregation properties have been investigated in aqueous environments only. Despite the relatively small size of the lipophilic part of LONs compared to the contour length of the nucleic acid polar head (typically $1.6 \mathrm{~nm}$ vs $8 \mathrm{~nm}$ for a 18 -mer cholesteryl LON for instance), ${ }^{53}$ most LONs can self-assemble in water as the hydrophobic effect can rapidly overcome coulombic repulsions. ${ }^{54}$ Yet, there is no definite answer as to the minimal size of the hydrophobic part that will lead to aggregation of the LON (a dodecane chain may be too short though). ${ }^{55}$ The types of aggregates formed from LONs are somewhat different from the pattern usually observed with classical surfactants. While classical single-chain and double-chain surfactants usually give predictable aggregated structures, no such simple trend is observed with LONs (Figure 4). 


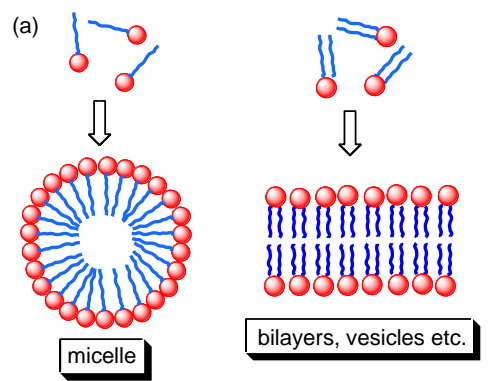

(b)

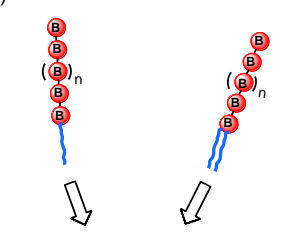

micelle, vesicles, bilayers and/or complex aggregates

Figure 4. Aggregation behavior of (a) traditional amphiphiles (single or double-chain) and (b) LONs (single or double-chain).

Hence, vesicles of single chain, ${ }^{56}$ and double-chain ${ }^{57}$ LONs have been characterized, just like micelles of single chain (mostly cholesterol-based), and double-chain ${ }^{58-60}$ LONs were reported. The hydrophobic motif in these examples (as well as in a vast majority of the LONs described in the literature) is attached at one of the extremity of the oligonucleotide sequence. Other constructs have been seldomly described and all gave micellar aggregates. ${ }^{61,62}$ Baglioni and coll. recently showed that cholesteryl-LON micelles coexist in water with smaller aggregates, the kinetics of the aggregation being slow. ${ }^{63}$

Many physical factors such as the ionic strength, temperature, $\mathrm{pH}$ etc. that influence the self assembly have not been investigated in details so far. One exception concerns the shape of the oligonucleotidic part. Gianneschi and coll. devised LON surfactants that were capable of reversibly switching from vesicles to micelles, ${ }^{57}$ or micelles to cylindrical assemblies ${ }^{61}$ upon base pairing to a complementary DNA and nuclease action respectively. The impact of the nucleic acid on the critical aggregation concentration (cac) of LON is important though largely unexplained. It must be borne in mind that unmodified oligonucleotides can have their own highly complex folding and equilibrium dynamic, ${ }^{64,65}$ whose impact on LON aggregates is virtually unknown. In fact, one can expect the cac of LON to increase due to electrostatic repulsions of the polyanionic DNA strands. Yet, while the classical DMPG has for instance a $\mathrm{cmc}$ value in the micromolar range, double-chain LONs self-associate at nanomolar concentrations, ${ }^{58,60}$ and the cmc seems ${ }^{66}$ to increase with the shortening of the nucleic acid. ${ }^{57}$ It is tempting here to ascribe this behavior to the proximity effects as those observed with DNA gold nanoparticle conjugates. ${ }^{67,68}$ Aliphatic chain aggregation may bring about nucleic acid segments in close proximity thus favoring and stabilizing partial base pairing and/or other tertiary structures between LONs with a concomitant decrease in the value of the cac. A thorough investigation is yet required to confirm this hypothesis. Other LONs derived from cholesterol (chol-LON) have cmc values in the low micromolar range. ${ }^{63,69}$

\subsection{LON incorporation into membranes.}

In many applications, LONs are used to tag vesicles and/or membranes. Hopefully, little quantities of LONs are then required so that many examples have been published lately. It must be first emphasized that there are comparatively far less reports on soft DNA-nanoparticles in comparison to hard ones (gold, quantum dots...) most probably because of robustness and handling issues. Yet, the dynamic nature of soft materials have been shown to be of great practical interest. For instance, fluid surfaces are potentially superior in DNA micro-array formats. In that case, oligonucleotide capture probes can rearrange to give homogeneous kinetics and thermodynamics upon target binding. Besides, Tan compared the recognition properties of DNA coupled to hard nanoparticles (nanotubes or gold) versus micelles of LONs. While all architectures bound the target protein at $4{ }^{\circ} \mathrm{C}$, the micelles alone were fluid enough to rearrange and keep the ability to bind to the target at $37^{\circ} \mathrm{C} .{ }^{60}$

The seminal work of Gosse and coll. ${ }^{58}$ highlighted the challenges associated with the incorporation of LON into membranes: 1) the LON must be capable of spontaneous insertion into the membrane, 2) the anchorage must be stable and 3) the LON must retain its ability to specifically bind its target. In practice, one has to find a compromise between: a weakly lipophilic LON that may desorb from the membrane and a very lipophilic LON that may form kinetically and/or thermodynamically stable self-assemblies which will not partition into the host membrane (Figure 5).

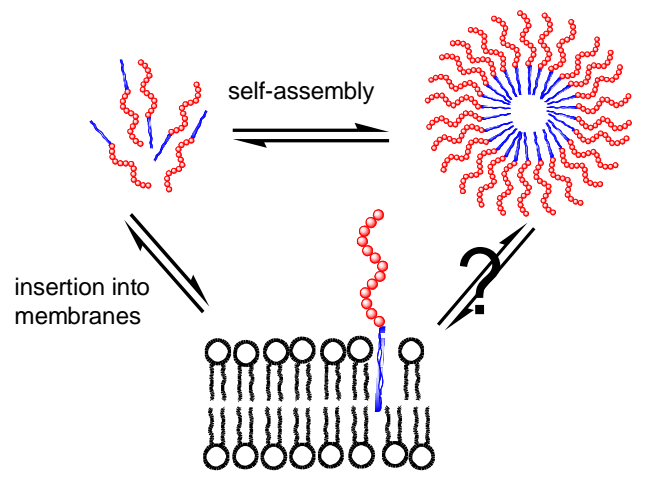

Figure 5. Competition between self-aggregation and insertion into membranes for LONs.

Höök confirmed that a single cholesteryl motif was not sufficient for the stable insertion of the LON into POPC membranes while a doubly-modified LON was. ${ }^{70}$ Similarly, chol-LONs partition between small unilamellar vesicles and a supported lipid bilayer. ${ }^{71}$ As an explanation, ${ }^{2} \mathrm{H}$ solid state NMR showed that in contrast to free cholesterol, the POPC membrane was not perturbed upon inclusion of chol-LONs, the cholesterol motif being anchored in a non-optimal geometry. ${ }^{72}$ Yet, the systematic desorption of cholLONs is still a debate. Baglioni found that his chol-LON was stably inserted into a supported lipid bilayer made of POPC, desorption being observed only when concentration of the LON exceeded $8 \mathrm{nM}$ or when hybridization to a complementary strand took place. ${ }^{53}$ On the contrary, there is no report of LONs that would self-associate so strongly as to prevent inclusion into bilayers. At best, such very lipophilic LONs thermodynamically partitions into self aggregates and bilayers. ${ }^{58}$

For the successful applications of LONs in biotechnological settings, LONs must first retain their ability to specifically bind the complementary oligonucleotide once inserted into the membrane. ${ }^{73}$ LON being anchored to soft matter, the DNA strands keep their ability to move and reorganize more or less freely in the two-dimensional plane of the membrane. This can constitute an advantage over most DNA-based micro-array formats, which overwhelmingly capitalize on hard supports and/or nanoparticles. Yet, the reports on the kinetics and/or thermodynamics of binding of unmodified nucleic acids to membrane bound LONs are scarce. 
As in the case of "hard" supports, ${ }^{74}$ the kinetics of binding were found to be highly dependent on the grafting density of the nucleic acid on the membrane, duplex formation being faster at lower coverage (before the mushroom to brush conformational change of the DNA strands on the surface). ${ }^{63}$ More interestingly, cooperativity was evidenced in the formation of the duplex with the LON-derived vesicle: the rate of hybridization appeared faster on the surface of the vesicle compared to the same unmodified duplex in the bulk. Anchorage of DNA to vesicles has obviously a great impact on duplex formation and most reported LON structures include a spacer in between the oligonucleotide sequence and the lipid to minimize surface effects. For instance, a PEG spacer was required for the observation of DNA duplex formation with a chol-LON inserted into the membrane of a negatively charged giant liposome. ${ }^{75}$ It is therefore not surprising that the thermodynamics of the annealing process with LONs were virtually unchanged when compared to bulk DNA in these cases. ${ }^{63,69}$ To the best of our knowledge, there is only one description of DNA pairing to membrane-bound and spacer-free LONs. Interestingly, the effect of the zwitterionic DOPC membrane was found to be negligible in comparison to the duplex in solution. ${ }^{32}$

Surprisingly, there are comparatively far more reports on the hybridization of complementary vesicles (each decorated with complementary strands). This was pioneered by Letsinger who devised an elegant strategy for the triggered aggregation of liposomes by target DNA pairing to a LON modified at both "ends" with a cholesterol residue (Figure 6). ${ }^{76}$

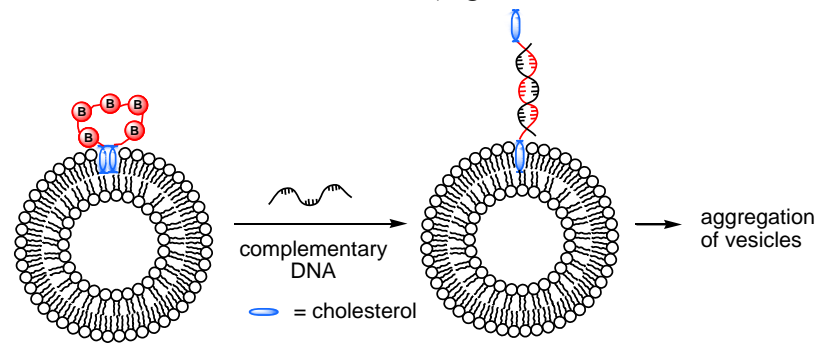

Figure 6. Base-pairing-triggered aggregation of liposomes, not drawn to scale.

At start, both cholesterol residues remain anchored to the surface of one single liposome. Upon binding the complementary DNA, one of the two cholesterol is expelled from the membrane and triggers the aggregation and sedimentation of bound-liposomes. The same principle was used by Vogel who further demonstrated that the melting profile of the assembly was very sharp with no partitioning of the LONs between membranes upon heating. ${ }^{77}$ Other LON-mediated vesicle aggregation examples have been published. ${ }^{78-81}$

A great deal of attention has been given to the tethering of vesicles to supported lipid bilayers and their docking once bound to the SLB, both mediated by base pairing between complementary LONs. The reason for the synthesis of such complex architectures stands from the fact that the properties (mobility, function) of membrane proteins are often altered by the solid support underlying the SLB. Biophysical investigations revealed that the probability of docking between complementary tethered vesicles is governed by the kinetics of hybridization: it increases with the number of LONs present at the surface, the number of repeats in the DNA sequence and the length of the linker in the LON sequence. $^{82}$ The diffusion of such tethered vesicles does not depend on the size of the tether and vesicle, but decreases with the number of anchors. ${ }^{83,84}$ The tether stiffness was also found important for an optimal diffusion of the vesicles on the SLB while the vesicles were unexpectedly "frozen" under low salt conditions. ${ }^{85}$

An interesting question underlying the aggregation of vesicles mediated by 2 complementary LONs concerns the critical number of duplexes that are necessary to hold two vesicles together. In fact, a single DNA duplex tether will be weakened as a result of Brownian motions of the two vesicles. As more LONs are present at the vesicle surface, LONs can rearrange to allow the accumulation of DNA duplexes in the binding site. This ultimately results in the stabilization of the vesicular assembly. ${ }^{86}$ Consequently, an average of 2.5 and 40 LONs per vesicle was found necessary to observe 1) detectable levels of vesicle association and 2) flocculation. ${ }^{86}$ The entropic loss upon formation of vesicular assemblies is less when the vesicles are tethered to a supported membrane: only a two-dimensional movement of the vesicles is then possible. As a result fewer anchors are necessary in that case and a single DNA anchor was sufficient to observe docking between vesicles. ${ }^{85,87,88}$

In all these examples, the lipid part of the complementary LONs was always attached at the same extremity of the DNA strand. Due to the antiparallel nature of the DNA recognition mode, the vesicles were separated by a rigid DNA duplex in the final aggregates. When the lipids are attached at the 3'-end of the DNA for one LON and at the 5'-end for the complementary LON, the two strands are forced to hybridize in a zipper-like fashion, forcing a close contact between the vesicles. This very simple design allowed the groups of Höök and Boxer to trigger the fusion of vesicles in a way that much resembles the mode of action of the complex SNARE proteins. ${ }^{89-92}$ If lipid mixing took place easily upon fusion (even in the inner leaflet) content mixing remained low in all cases (17\% at best). The number of LONs on the surface and the number of repeats in the DNA sequence were important parameters to favor the fusion process..$^{90,91}$ Besides, if the presence of a spacer favors docking of vesicles, it decreases fusion efficiency. Finally, the distance between vesicles is truly the key factor as fusion was still observed upon "regular" (no-zipper-like hybridization, oleic acid being present at the 5'-end of both LONs) base pairing between 2 short 8 -mer LONs (no fusion was observed with 24-mer analogs). ${ }^{93}$

As pointed out by Vanderlick and coll., melting temperatures of DNA-linked nanoparticle assemblies cannot be understood directly from models of the thermodynamics of single DNA strands in solution. Among the important differences, there are: 1) entropy cost of tethering one end of single stranded DNA to the vesicle, 2) entropy loss of the particles once bound in the aggregate as discussed before, 3) locally increased concentration and orientational restriction of LON in the membrane, 4) influence of inter-vesicle interactions. ${ }^{81}$ While point 2 clearly disfavors duplex formation, it is counterbalanced by points 3 and possibly 4 . In fact, melting temperature of DNA duplexes in the vesicular assembly was lowest when the vesicles were negatively charged and highest when the vesicles were diluted with a positively charged lipid revealing the importance of electrostatic interactions in the stabilization of the assembly. ${ }^{81}$ Interestingly, complementary 
bound vesicles usually melt with a higher Tm than bulk DNA duplexes and with a sharper melting profile (the melting occur over a short temperature interval) ${ }^{77}$ reminiscent of what is observed with gold nanoparticle-DNA conjugates. The higher local concentration and sharing of the ion cloud in proximal DNA duplexes, the so-called proximity effect, ${ }^{68}$ are usually inferred to explain this observation. When the first duplex melts, the counterions are split into two between the two dissociated species thus weakening the proximal duplexes that share the same ion cloud. Indeed, it was shown that the increased Tm observed with LONs does not solely result from a local increase in the effective DNA concentration on the vesicle surface compared to bulk DNA. An additional undisclosed parameter was necessary to explain the difference between the model and the experimental data. ${ }^{81}$ This additional parameter could well be related to the sharing of the ion cloud.

So far, the lipophilic anchors have been regarded as relatively unspecific "glues" that would stick to any hydrophobic environments. Yet, the different lipidic components of natural membrane like plasma membrane or artificial analogs are not homogeneously distributed in the membrane. This leads to lipid domains and rafts with different lipid composition and with different biophysical properties. Arbuzova and coll. have been active in the design of LONs whose lipid part specifically bind in liquid ordered or liquid disordered domains. ${ }^{72,94-96}$ Interestingly, they were capable of switching reversibly with temperature the segregation of an a-tocopherol LON and a PNA derivatized with palmitic acid on the surface of CHO-K1 cells. ${ }^{96}$

The modification of live cell surface with LON is obviously very exciting to tag and/or impart new properties to cultured cells. This issue was addressed lately with the modification of different cell lines with stearoyl- or palmitoyl-LON. It was for instance possible to mediate the docking of floating HL60 to adherent A431 epitheliocytes with complementary 70 -mer LONs. ${ }^{97}$ The LON was shown to be anchored to the cell surface very rapidly but not surprisingly, the effect remained short as the LON is progressively taken up by the cell, most probably via endocytosis. Just like with artificial membranes, ${ }^{82}$ the kinetics of docking between cells was found to depend on the surface DNA density as well as the number of repeats within the DNA sequence. ${ }^{98}$

\subsection{Recent applications of LONs}

LONs have been utilized in a broad range of applications summarized in our previous perspective article. ${ }^{4}$ The field of applications of LONs basically ranges from RNA interference to detection, quantification and purification of nucleic acids.

Höök has developed a sandwich assay for the detection of target nucleic acids using TOF secondary ion mass spectrometry. The limit of detection of traditional mass analysis in the nanomolar range was decreased to detect picomolar target concentrations using the detection of the lipid component of the vesicles once target probe is sandwiched between the silica solid support and the LON inserted into the vesicle. The recognition of one single target DNA induces the binding of the total lipid payload of one vesicle thus amplifying the mass signal. Multiplexing analyses were made possible playing around the lipid composition of the vesicles and the sequence of the embarked LON. ${ }^{88}$ Using a similar protocol, the authors could follow the kinetics of base pairing of the vesicles to the solid support. They showed this format to be far superior for the detection of single base mismatches over traditional techniques that usually rely on end-point affinity measurements with a captured probe. ${ }^{99,100}$

DNA origami construction on the surface of POPC liposomes with LON as a primary building block was also demonstrated. Interestingly, the tedious heating-and-cooling protocol for the build-up of DNA origami was not necessary here. DNA hexagons were assembled easily at room temperature on the vesicle membrane. ${ }^{101}$

Finally, the properties of LON to self-assemble into micelles have been successfully applied in two recent papers. Cornelissen and Herrmann were able to replace the natural viral RNA for LON micelles to template the assembly of the cowpea chlorotic mottle virus capsid. ${ }^{62}$ This artificial virus was in turn capable of loading 1) lipophilic molecules in the micellar core of the particle and 2) extra chemicals covalently linked to a complementary DNA. In the second application, Tan synthesized an aptameric LON. Aptamer are the nucleic acid versions of antibodies. They can be selected against any given target from a random population of DNA or RNA molecules by a process called SELEX. The aptamer raised against immunoglobulin were derivatized with 2 stearoyl chains. Very interestingly, this LON has unique recognition properties over the underivatized aptamer in the sense that the LON could still recognize its target in the presence of blood in contrast to the unmodified aptamer. Multivalency of the micelles formed from this LON was hypothesized to explain this observation (estimated 1000 copies of LON per micelle) in addition to the lipidic anchor that can stabilize the LON micelle in the vicinity of the membranebound protein target. ${ }^{60}$

\section{Biomedical applications}

Successful biomedical applications based on the administration of LONs in vivo are mostly dedicated to the field of RNA interference (RNAi).

Following the discovery of RNAi as a means to silence expression of specific genes involved in disease, new gene therapy strategies were developed. ${ }^{102}$ The two key actors of the RNAi pathway, which are the targets of new RNAi-based therapeutics are small interfering RNA (siRNAs) ${ }^{103}$ and microRNA (miRNA). ${ }^{104}$ Despite similarities in their silencing pathways, including a common loading into a protein complex called the RNA induced silencing complex (RISC), the modes of action of these molecules present differences. Both siRNA and miRNA target mRNA, acting as posttranscriptional regulators. The success of siRNA and miRNAbased therapeutics in in vitro studies has recently prompted the development of in vivo investigations. Nevertheless in vivo delivery remains one of the major obstacle of this therapeutic strategy stimulating the active research of vector-based delivery system. In this context LONs have successfully been used to silence gene expression in mice and in non-human primates. ${ }^{105-108}$ As anticipated by physico-chemical investigations, including selfassembly capabilities of LONs and their incorporation into membranes (see previous section), the hydrophobic part impacts the in vivo distribution and cell penetration. A great deal of attention should be given to the selection of their hydrophobic part thanks to handling biomedical issues and more particularly to targeted tissues. It must be emphasized that LONs are mainly 
exploited in the context of liver targeting, the rationale being the potential interaction of LONs with serum lipoprotein and the following cell penetration through receptor-mediated endocytosis. The delivery moiety of LONs is expected to facilitate in vivo cell penetration and distribution. Nevertheless, the anchoring of LONs into cellular membranes or endosomal compartments could alter the biological activities of the oligonucleotide sequences. LON structures should be optimized to i) favour cell penetration, ii) retain their specific loading by RISC and iii) bind to the mRNA target.

\subsection{Cholesterol}

To date, studies on LON biomedical applications capitalize on cholesterol as the hydrophobic moiety. LONs conjugated to cholesterol exhibit markedly improved cellular uptake in vitro and in the liver as well as elicits robust RNAi, which results in silencing of endogenous genes in vivo. Cholesterol has been demonstrated to interact with lipoproteins favoring their uptake via lipoproteinreceptor mediated pathways. The presence of these receptors on cell membranes is strikingly correlated to the penetration of cholesterol-LON. Cholesterol derivatives are also described to penetrate cells through endocytosis mechanisms after their anchoring in hydrophobic cellular membranes.

Concerning the miRNA field, the inhibition of overexpressed miRNA by the use of cholesterol-LON known as antagomirs has been performed. ${ }^{10,109}$ Antagomirs feature an oligonucleotide part with a sequence complementary to the targeted overexpressed miRNA and a cholesterol part at the $3^{\prime}$ end. The linker between cholesterol and the oligonucleotide is a commercially available hydroxyprolinol support (Figure 7A). Firstly described for the inhibition of miR-122 in the liver, ${ }^{10}$ the recent use of antagomirs for the silencing of miR-10b in a mouse mammary tumor model augurs well for the development of new miRNA-based therapeutics. ${ }^{105}$ The systemic injection of $50 \mathrm{mg} / \mathrm{kg}$ of antagomirs induces gene silencing in mice and resulted in the inhibition of metastasis in highly metastatic cancer. This study provides a proofof-principle that cholesterol-LON antagomirs can be efficiently delivered to rapidly growing tumor cells in vivo and can prevent metastasis formation.

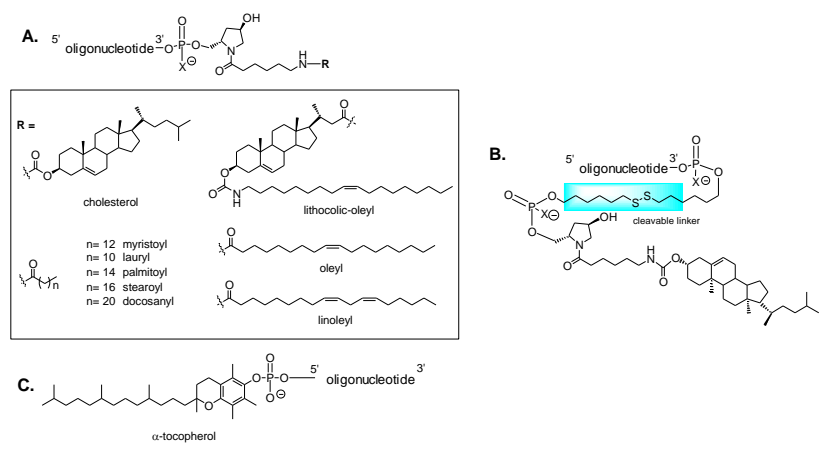

Figure 7. Examples of LONs for biomedical applications

In the case of siRNA, cholesterol was covalently linked to the $3^{\prime}$ extremity of the passenger strand of the double stranded siRNA by using a similar strategy based on a hydroxyprolinol support (Figure 7A.). ${ }^{102}$ In this study, cholesterol-conjugated siRNA targeting apolipoprotein $\mathrm{B}$ (apoB) was injected in mice to modulate apoB expression. After a systemic injection, the suppression of apoB mRNA and a decrease in the levels of plasma apoB and serum cholesterol was obtained. The interactions between cholesterol moiety of LONs and high density lipoprotein (HDL) as well as low density lipoprotein (LDL) were observed. More precisely, a preassembly between cholesterol-siRNA and HDL was shown to promote a 8 to 15 more effective apoB gene silencing in vivo than similar dosing paradigm of free cholesterol-siRNA. This result reinforces the principle of lipoprotein-receptor mediated endocytosis for cholesterol-LON cell penetration.

The detection of LDL receptors in brain prompted the development of cholesterol-conjugated siRNA dedicated to penetrate neurons in vivo. Three recent studies describing the therapeutic potential of cholesterol-siRNA for central nervous system (CNS) disorders are reported. They all exploited the hydroxyprolinol strategy to attach the cholesterol moiety to the 3 ' end of the siRNA passenger strand (Figure 7A). The first one concerns the huntington's disease, a neurodegenerative disorder which can be addressed via RNA silencing. ${ }^{6}$ A local injection in neurons of mouse CNS demonstrated the uptake of cholesterol-siRNA by neurons and their silencing efficiency. The second study describes the inhibition of an oligodendrocyte-specific gene in the rat as well as in non-human primate CNS after a direct CNS injection. ${ }^{108}$ The third one is also related to the silencing of a gene specifically expressed in the CNS by oligodendrocytes. ${ }^{106}$ In this case the advantage of using cleavable disulfide linker was demonstrated for improving the inhibition (by a factor of 2) of this specific gene in oligodendrocytes in vivo after a direct intraparenchymal CNS infusion (Figure 7B). Altogether, these results suggest that the uptake of cholesterol-siRNA in neuronal cells may occur via lipoprotein receptors.

\subsection{Lipids}

siRNA targeting apoliprotein B (apoB) were also conjugated to lipids such as bile acids and long chain fatty acids (Figure 7A). ${ }^{110}$ Lipids were linked to the 3 ' end of the siRNA passenger strand through an hydroxyprolinol bridge. After intravenous injection in mice, their ability to modulate apoB expression was investigated by measuring apoB transcript levels.

The major observation concerns the impact of lipids on the gene silencing. Shorter chains such as lauroyl, myristoyl and palmitoyl lead to an absence of effect on apoB transcript levels compared to longer chain such as steroyl, docanosyl and lithocholic-oleyl lipids. It was further demonstrated that such behaviour could be ascribed to the different affinities of the lipids for lipoprotein particles. For instance, LONs possessing lower hydrophobicity exhibit a poor affinity for lipoproteins compared to larger fatty acid chains or lithocholic-oleyl lipids. In summary, this study highlights the role of serum lipoprotein in the control of LON uptake in the liver. ${ }^{110}$

\subsection{Lipophilic small molecules}

The use of a fat-soluble natural molecule such as $\alpha$-tocopherol was also envisioned in the context of a 27/29 mers siRNA designed to silence apoB (Figure 7C). ${ }^{111}$ Tocopherol was covalently attached to the 5' end of the siRNA guide strand. In cells, this long siRNA was submitted to a maturation step involving the enzyme DICER, which leads to the release of tocopherol and the generation of an active 21 mers siRNA. The impact the $\alpha$-tocopherol conjugation 
on the silencing ability was studied in cultured cells of mouse hepatocellular carcinoma (Hepa 1-6) in vivo after intravenous injection to mice. For that purpose, the apoB silencing effects of siRNA linked to tocopherol were compared to those obtained with siRNA linked to cholesterol, after an intravenous injection of 2 $\mathrm{mg} / \mathrm{Kg}$ in mice. Surprisingly, in these conditions, the reduction of apoB mRNA was only detected with tocopherol-siRNA. The differences of efficiency between cholesterol and tocopherol siRNA can be attributed to their different affinities for serum proteins, tocopherol interacting preferentially to tocopherolassociated proteins such as SEC14L2, SEC14L3, SEC14L4 and afamin. This is reinforced by in vitro experiments in which serum was essential to promote silencing effects, emphasizing the need for interactions between tocopherol and serum proteins to penetrate hepatoma cells and the potential involvement of lipoprotein-receptor mediated pathways as cell penetrating mechanism. Interestingly, tocopherol conjugated siRNA did not induce IFN- $\alpha$ in serum and IFN- $\beta$ mRNA in the liver offering a very promising and the safe system for liver drug delivery.

\section{Conclusion}

Owning to their intrinsic hybrid molecular structures, LONs have to rely on the unique combination of the hydrophilic ON polar heads with lipid moieties. The examples of LONs presented in this contribution show that different synthetic strategies allow a facile access to LONs structures with hydrophobic modification(s) basically anywhere in the oligonucleotide sequences. Interestingly, the current physicochemical studies realized by several academic groups supports that "smart" LONs cannot be consider as simple "dummy" surfactants in aquous media. The multitude of assemblies observed, including micelles, vesicles like aggregates, cylindrical systems or complex nanoparticles shows that the aggregation behaviors of these amphiphiles is more than complex and depend on multiple parameters. Indeed, it is still difficult to predict the influence of different environmental and molecular criteria on the aggregation phenomena. Experimental conditions such as the ionic strength, $\mathrm{pH}$ or temperature, or the nature of the hydrophophic moiety and its position on the $\mathrm{ON}$ sequences, the sequences themselves are all undubitably playing a role in the aggregation of LONs. One key challenge in the next few years will be therefore to determine the impact of these non-structural and molecular parameters on the self-assemblies. Likewise, the cohabitations of LONs with synthetic and/or biological membranes are just starting to receive attention. A clear understanding of the relationships occurring between these partners is definitively required for applications involving LONs and cells, for example.

It is worth extending the contribution of the knowledge on LONs into the larger perspective of biomedical applications. Together with the intellectual beauty of investigations that range from molecular and supramolecular engineering to biomedical applications, this research has already provided practical tools to improve the delivery in cellulo of active sequences by, for example, avoiding the use of some transfecting reagents recognized as potentially toxic (i.e., polycationic artificial carrier). The vectorization of new biologically relevant sequences, including anti-miR, antisense,
siRNA or aptamers will be certainly a central preoccupation of researchers for the future applications of LONs.

The history of this research is still at the beginning and we can safely state that the route is surely paved of remarkable future achievements.

\section{Acknowledgment}

P.B. acknowledges financial support from the Army Research Office. This work has been supported by the French National Agency (ANR) in the frame of its programme PNANO (project NANAN, ${ }^{\circ}$ ANR-08-NANO-028).

\section{References}

1 H. Liu, Z. Zhu, H. Kang, Y. Wu, K. Sefan, and W. Tan, Chem. Eur. J., 2010, 16, 3791.

2 M. P. Thompson, M.-P. Chien, T.-H. Ku, A. M. Rush, and N. C. Gianneschi, Nano Lett., 2010, 10, 2690.

3 F. Gambinossi, M. Banchelli, A. Durand, D. Berti, T. Brown, G. Caminati, and P. Baglioni, J. Phys. Chem. B., 2010, 114, 7338.

4 A. Gissot, M. Camplo, M. W. Grinstaff, and P. Barthélémy, Org. Biomol. Chem., 2008, 6, 1324.

5 C. Mao, T. H. LaBean, J. H. Reif, and N. C. Seeman, Nature, 2000, 407, 493.

6 N. Bowden, A. Terfort, J. Carbeck, and G. M. Whitesides, Science, 1997, 276, 233.

7 M. Lee, C.-J. Jang, and J.-H. Ryu, J. Am. Chem. Soc., 2004, 126, 8082.

8 A. Petitjean, R. G. Khoury, N. Kyritsakas, and J.-M. Lehn, J. Am. Chem. Soc., 2004, 126, 6637.

9 G. M. Whitesides, J. P. Mathias, and C. T. Seto, Science, 1991, 254, 1312.

10 J. Krützfeldt, N. Rajewsky, R. Braich, K. G. Rajeev, T. Tuschl, M. Manoharan, and M. Stoffel, Nature, 2005, 438, 685.

11 G. Godeau, H. Arnion, C. Brun, C. Staedel, and P. Barthélémy, Med. Chem. Commun., 2010, 1, 76.

12 F. E. Alemdaroglu and A. Herrmann, Org. Biomol. Chem., 2007, 5, 1311.

13 H. Lönnberg, Bioconjug. Chem., 2009, 20, 1065.

14 M. Manoharan, K. L. Tivel, and P. D. Cook, Tetrahedron Lett., 1995, 36, 3651 .

15 C. MacKellar, D. Graham, D. W. Will, S. Burgess, and T. Brown, Nucleic Acids Res., 1992, 20, 3411.

16 R. L. Letsinger, G. Zhang, D. K. Sun, T. Ikeuchi, and P. S. Sarin, Proc. Natl. Acad. Sci. U.S.A., 1989, 86, 6553.

17 J. D’Onofrio, D. Montesarchio, L. De Napoli, and G. Di Fabio, Org. Lett., 2005, 7, 4927.

18 D. A. Stetsenko and M. J. Gait, Bioconjug. Chem., 2001, 12, 576.

19 C. J. Marasco Jr., N. J. Angelino, B. Paul, and B. J. Dolnick, Tetrahedron Lett., 1994, 35, 3029.

20 Y. Ueno, K. Kawada, T. Naito, A. Shibata, K. Yoshikawa, H.-S. Kim, Y. Wataya, and Y. Kitade, Org. Biomol. Chem., 2008, 16, 7698.

21 M. Manoharan, Antisense Nucleic Acids Drug Dev., 2002, 12, 103.

22 A. Rait, K. Pirollo, D. W. Will, A. Peyman, V. Rait, E. Uhlmann, and E. H. Chang, Bioconjug. Chem., 2000, 11, 153.

23 I. Habus, Q. Zhao, and S. Agrawal, Bioconjug. Chem., 1995, 6, 327.

24 H. Vu, T. S. Hill, and K. Jayaraman, Bioconjug. Chem., 1994, 5, 666. 
25 H. Vu, P. Singh, L. Lewis, J. G. Zendegui, and K. Jayaraman, Nucleosides Nucleotides, 1993, 12, 853.

26 D. W. Will and T. Brown, Tetrahedron Lett., 1992, 33, 2729.

27 M. W. Reed, A. D. Adams, J. S. Nelson, and R. B. Meyer Jr., Bioconjug. Chem., 1991, 2, 217.

28 S. Matysiak, R. Frank, and W. Pfleiderer, Nucleosides Nucleotides, 1997, 16, 855.

29 M. W. Reed, E. A. Lukhtanov, V. V. Gorn, D. D. Lucas, J. H. Zhou, S. B. Pai, Y.-C. Cheng, and R. B. Meyer Jr., J. Med. Chem., 1995, 38, 4587.

30 R. G. Shea, J. C. Marsters, and N. Bischofberger, Nucleic Acids Res., 1990, 18, 3777.

31 T. J. Lehmann and J. W. Engels, Bioorg. Med. Chem., 2001, 9, 1827.

32 A. Gissot, C. Di Primo, I. Bestel, G. Giannone, H. Chapuis, and P. Barthélémy, Chem. Commun., 2008, 5550.

33 R. Huisgen, 1,3-Dipolar Cycloaddition Chemistry, A. Padwa, NewYork, Wiley., 1984, vol. 1.

34 V. V. Rostovtsev, L. G. Green, V. V. Fokin, and K. B. Sharpless, Angew. Chem., Int. Ed., 2002, 41, 2596.

35 G. Godeau, C. Staedel, and P. Barthélémy, J. Med. Chem., 2008, 51, 4374.

36 S. V. Vinogradov, T. Le Doan, and C. Hélène, Tetrahedron Lett., 1995, 36, 2493.

37 F. Ramirez, S. B. Mandal, and J. F. Marecek, J. Am. Chem. Soc., 1982, 104, 5483.

38 M. Acedo, G. Tarrason, J. Piulats, M. Mann, M. Wilm, and R. Eritja, Bioorg. Med. Chem. Lett., 1995, 5, 1577.

39 G. Tosquellas, K. Alvarez, C. Dell'Aquila, F. Morvan, J.-J. Vasseur, J.-L. Imbach, and B. Rayner, Nucleic Acids Res., 1998, 26, 2069.

40 N. N. Polushin and J. S. Cohen, Nucleic Acids Res., 1994, 22, 5492.

41 A. Guzaev and M. Manoharan, Bioorg. Med. Chem. Lett., 1998, 8, 3671.

42 A. Kobylańska, A. Okruszek, and W. J. Stec, Nucleosides Nucleotides, 1998, 17, 1977.

43 A. Durand and T. Brown, Nucleosides Nucleotides Nucleic Acid, 2007, 26, 785 .

44 A. Kurz, A. Bunge, A.-K. Windeck, M. Rost, W. Flasche, A. Arbuzova, D. Strohbach, S. Müller, J. Liebscher, D. Huster, and A. Herrmann, Angew. Chem., Int. Ed., 2006, 45, 4440.

45 J. M. Tomkins, K. J. Barnes, A. J. Blacker, W. J. Watkins, and C. Abell, Tetrahedron Lett., 1997, 38, 691.

46 T. Bryld and C. Lomholt, Nucleosides Nucleotides Nucleic Acid, 2007, 26, 1645.

47 H. Chapuis, L. Bui, I. Bestel, and P. Barthélémy, Tetrahedron Lett., 2008, 49, 6838.

48 A. Guzaev and H. Lönnberg, Tetrahedron, 1999, 55, 9101.

49 S. J. Kim, E.-K. Bang, H. J. Kwon, J. S. Shim, and B. H. Kim, ChemBioChem, 2004, 5, 1517.

50 A. V. Kabanov, S. V. Vinogradov, A. V. Ovcharenko, A. V. Krivonos, N. S. Melik-Nubarov, V. I. Kiselev, and E. S. Severin, FEBS Lett., 1990, 259, 327.

51 M. Manoharan, L. K. Johnson, K. L. Tivel, R. H. Springer, and P. D. Cook, Bioorg. Med. Chem. Lett., 1993, 3, 2765.

52 S. M. Gryaznov and D. H. Lloyd, Nucleic Acids Res., 1993, 21, 5909.

53 F. Gambinossi, M. Banchelli, A. Durand, D. Berti, T. Brown, G. Caminati, and P. Baglioni, J. Phys. Chem. B, 2010, 114, 7338.
54 C. Tribet and F. Vial, Soft Matter, 2008, 4, 68

55 B. M. Laing, L. Barrow-Laing, M. Harrington, E. C. Long, and D. E. Bergstrom, Bioconjug. Chem., 2010, 21, 1537.

56 P. M. Dentinger, B. A. Simmons, E. Cruz, and M. Sprague, Langmuir, 2006, 22, 2935.

57 M. P. Thompson, M.-P. Chien, T.-H. Ku, A. M. Rush, and N. C. Gianneschi, Nano Lett., 2010, 10, 2690.

58 C. Gosse, A. Boutorine, I. Aujard, M. Chami, A. Kononov, E. CognéLaage, J.-F. Allemand, J. Li, and L. Jullien, J. Phys. Chem. B, 2004, 108, 6485.

59 H. Liu, Z. Zhu, H. Kang, Y. Wu, K. Sefan, and W. Tan, Chem. Eur. J., 2010, 16, 3791.

60 Y. Wu, K. Sefah, H. Liu, R. Wang, and W. Tan, Proc. Natl. Acad. Sci. U.S.A., 2010, 107, 5 .

61 M.-P. Chien, A. M. Rush, M. P. Thompson, and N. C. Gianneschi, Angew. Chem. Int. Ed., 2010, 49, 5076.

62 M. Kwak, I. J. Minten, D.-M. Anaya, A. J. Musser, M. Brasch, R. J. M. Nolte, K. Müllen, J. J. L. M. Cornelissen, and A. Herrmann, J. Am. Chem. Soc., 2010, 132, 7834.

63 M. Banchelli, F. Betti, D. Berti, G. Caminati, F. B. Bombelli, T. Brown, L. M. Wilhelmsson, B. Nordén, and P. Baglioni, J. Phys. Chem. B, 2008, 112, 10942.

64 G. Bokinsky and X. Zhuang, Acc. Chem. Res., 2005, 38, 566.

65 J. Zhou, S. K. Gregurick, S. Krueger, and F. P. Schwarz, Biophys. J., 2006, 90, 544.

66 The cmc values for double-chain LONs are scarse in the literature and were measured under different conditions. A more in-depth investigation is necessary to ascertain this trend.

67 S. J. Hurst, H. D. Hill, and C. A. Mirkin, J. Am. Chem. Soc., 2008, 130, 12192.

68 I. Eryazici, T. R. Prytkova, G. C. Schatz, and S. T. Nguyen, J. Am. Chem. Soc., 2010, 132, 17068.

69 M. Banchelli, F. Gambinossi, A. Durand, G. Caminati, T. Brown, D. Berti, and P. Baglioni, J. Phys. Chem. B, 2010, 114, 7348.

70 I. Pfeiffer and F. Höök, J. Am. Chem. Soc., 2004, 126, 10224.

71 I. Pfeiffer and F. Höök, Anal. Chem., 2006, 78, 7493.

72 A. Bunge, M. Loew, P. Pescador, A. Arbuzova, N. Brodersen, J. Kang, L. Dähne, J. Liebscher, A. Herrmann, G. Stengel, and D. Huster, J. Phys. Chem. B, 2009, 113, 16425.

73 For Tms of LONs which are not inserted into membranes, the reader is referred to ref 4.

74 A. W. Peterson, R. J. Heaton, and R. M. Georgiadis, Nucleic Acids Res., 2001, 29, 5163 .

75 K.-ichiroh Shohda, T. Toyota, T. Yomo, and T. Sugawara, ChemBioChem, 2003, 4, 800.

76 G. Zhang, F. Farooqui, O. Kinstler, and R. L. Letsinger, Tet. Lett., 1996, 37, 6243.

77 U. Jakobsen, A. C. Simonsen, and S. Vogel, J. Am. Chem. Soc., 2008, 130, 10462.

78 N. Maru, K.-ichiroh Shohda, and T. Sugawara, Nucleic Acids Symp. Ser., 2004, 48, 95 .

79 A. Granéli, M. Edvardsson, and F. Höök, ChemPhysChem, 2004, 5, 729.

80 M. Loew, J. Kang, L. Dähne, R. Hendus-Altenburger, O. Kaczmarek, J. Liebscher, D. Huster, K. Ludwig, C. Böttcher, A. Herrmann, and A. Arbuzova, Small, 2009, 5, 320. 
81 P. A. Beales and T. K. Vanderlick, Biophys. J., 2009, 96, 1554.

82 Y.-H. M. Chan, P. Lenz, and S. G. Boxer, Proc. Natl. Acad. Sci. U.S.A., 2007, 104, 18913

83 C. Yoshina-Ishii, Y.-H. M. Chan, J. M. Johnson, L. A. Kung, P. Lenz, and S. G. Boxer, Langmuir, 2006, 22, 5682.

84 J. J. Benkoski and F. Höök, J. Phys. Chem. B, 2005, 109, 9773.

85 B. Van Lengerich, R. J. Rawle, and S. G. Boxer, Langmuir, 2010, 26, 8666.

86 P. A. Beales and T. K. Vanderlick, J. Phys. Chem. A, 2007, 111, 12372.

87 M. M. Lozano, C. D. Starkel, and M. L. Longo, Langmuir, 2010, 26, 8517.

88 A. Gunnarsson, P. Sjövall, and F. Höök, Nano Lett., 2010, 10, 732.

89 G. Stengel, R. Zahn, and F. Höök, J. Am. Chem. Soc., 2007, 129, 9584.

90 G. Stengel, L. Simonsson, R. A. Campbell, and F. Höök, J. Phys. Chem. B, 2008, 112, 8264.

91 Y.-H. M. Chan, B. van Lengerich, and S. G. Boxer, Proc. Natl. Acad. Sci. U.S.A., 2009, 106, 979 .

92 Y.-H. M. Chan, B. van Lengerich, and S. G. Boxer, Biointerphases, 2008, 3, FA17.

93 T. Maruyama, H. Yamamura, M. Hiraki, Y. Kemori, H. Takata, and M. Goto, Colloids Surf., B, 2008, 66, 119.

94 A. Bunge, A. Kurz, A.-K. Windeck, T. Korte, W. Flasche, J. Liebscher, A. Herrmann, and D. Huster, Langmuir, 2007, 23, 4455.

95 P. A. Beales and T. K. Vanderlick, J. Phys. Chem. B, 2009, 113, 13678.

96 M. Loew, R. Springer, S. Scolari, F. Altenbrunn, O. Seitz, J. Liebscher, D. Huster, A. Herrmann, and A. Arbuzova, J. Am. Chem. Soc., 2010, 132, 16066.

97 G. G. Borisenko, M. A. Zaitseva, A. N. Chuvilin, and G. E. Pozmogova, Nucleic Acids Res., 2009, 37, e28.

98 Z. J. Gartner and C. R. Bertozzi, Proc. Natl. Acad. Sci. U.S.A., 2009, 106, 4606 .

99 A. Gunnarsson, P. Jonsson, R. Marie, J. O. Tegenfeldt, and F. Hook, Nano Lett., 2008, 8, 183.
100 A. Gunnarsson, P. Jönsson, V. P. Zhdanov, and F. Höök, Nucleic Acids Res., 2009, 37, e99.

101 F. Baldelli Bombelli, F. Betti, F. Gambinossi, G. Caminati, T. Brown, P. Baglioni, and D. Berti, Soft Matter, 2009, 5, 1639.

102 C. V. Pecot, G. A. Calin, R. L. Coleman, G. Lopez-Berestein, and A. K. Sood, Nat. Rev. Cancer, 2011, 11, 59.

103 D. Grimm, Adv. Drug Delivery Rev., 2009, 61, 672.

104 R. Garzon, G. Marcucci, and C. M. Croce, Nat. Rev. Drug Discovery, $2010,9,775$.

105 L. Ma, F. Reinhardt, E. Pan, J. Soutschek, B. Bhat, E. G. Marcusson, J. Teruya-Feldstein, G. W. Bell, and R. A. Weinberg, Nat. Biotechnol., 2010, 28, 341 .

106 Q. Chen, D. Butler, W. Querbes, R. K. Pandey, P. Ge, M. A. Maier, L. Zhang, K. G. Rajeev, L. Nechev, V. Kotelianski, M. Manoharan, and D. W. Y. Sah, J. Controlled Release, 2010, 144, 227.

107 M. DiFiglia, M. Sena-Esteves, K. Chase, E. Sapp, E. Pfister, M. Sass, J. Yoder, P. Reeves, R. K. Pandey, K. G. Rajeev, M. Manoharan, D. W. Y. Sah, P. D. Zamore, and N. Aronin, Proc. Natl. Acad. Sci. U.S.A., 2007, 104, 17204.

108 W. Querbes, P. Ge, W. Zhang, Y. Fan, J. Costigan, K. Charisse, M. Maier, L. Nechev, M. Manoharan, V. Kotelianski, and D. W. Y. Sah, Oligonucleotides, 2009, 19, 23.

109 J. Krützfeldt, S. Kuwajima, R. Braich, K. G. Rajeev, J. Pena, T. Tuschl, M. Manoharan, and M. Stoffel, Nucleic Acids Res., 2007, 35, 2885.

110 C. Wolfrum, S. Shi, K. N. Jayaprakash, M. Jayaraman, G. Wang, R. K. Pandey, K. G. Rajeev, T. Nakayama, K. Charrise, E. M. Ndungo, T. Zimmermann, V. Koteliansky, M. Manoharan, and M. Stoffel, Nat. Biotechnol., 2007, 25, 1149.

111 K. Nishina, T. Unno, Y. Uno, T. Kubodera, T. Kanouchi, H. Mizusawa, and T. Yokota, Mol. Ther., 2008, 16, 734. 